\title{
Effects of Controller and Nonuniform Temperature Profile on the Onset of Rayleigh-Bénard-Marangoni Electroconvection in a Micropolar Fluid
}

\author{
H. M. Azmi and R. Idris \\ School of Informatics and Applied Mathematics, Universiti Malaysia Terengganu, 21030 Kuala Terengganu, Terengganu, Malaysia \\ Correspondence should be addressed to R. Idris; ruwaidiah@umt.edu.my
}

Received 5 February 2014; Accepted 11 May 2014; Published 16 June 2014

Academic Editor: Li Weili

Copyright (C) 2014 H. M. Azmi and R. Idris. This is an open access article distributed under the Creative Commons Attribution License, which permits unrestricted use, distribution, and reproduction in any medium, provided the original work is properly cited.

\begin{abstract}
Linear stability analysis is performed to study the effects of nonuniform basic temperature gradients on the onset of RayleighBénard-Marangoni electroconvection in a dielectric Eringen's micropolar fluid by using the Galerkin technique. In the case of Rayleigh-Bénard-Marangoni convection, the eigenvalues are obtained for an upper free/adiabatic and lower rigid/isothermal boundaries. The influence of various parameters has been analysed. Three nonuniform basic temperature profiles are considered and their comparative influence on onset of convection is discussed. Different values of feedback control and electric number are added up to examine whether their presence will enhance or delay the onset of electroconvection.
\end{abstract}

\section{Introduction}

Electrohydrodynamics is a branch of fluid mechanics which involved the effects of electrical forces. It is the study of the dynamics of electrically charged fluids involving the movements of ionized particles or molecules and their interactions with electric fields and the surrounding fluids. In dielectric media, hydrostatic pressure (or motion) is created by electrostatic fields. When media are fluids, a flow is produced. Generally, this phenomenon is related to the direct conversion of electrical energy into kinetic energy and vice versa. An electric field which is generated by electrically charged particles as well as time-varying magnetic field is the onset of natural convection. Magnetic effects will be dominant in highly conductive fluids. Ironically, electric effects will control the motion in dielectric fluids with such low values of the conductivity. The effect of electric fields on the liquid flow is directly converted into the kinetic energy [1].

Evolution of convection in a fluid has been greatly studied by many authors; see [2-8]. Convection is the transfer of thermal energy from one particle to another by the motion of fluids such as liquids or gases in a media. In general, convection can be divided into two which are natural convection and force convection. An example of natural convection is electroconvection. Electroconvection is the movements of fluid in an electric field. Many authors have studied the onset of convection in a dielectric field layer such as Roberts [2], Char and Chiang [3], Douiebe et al. [9], and El-Sayed [10].

In 1900, Bénard has observed a hexagonal pattern of convection cells below after heating some wax in a hot metal dish above some critical temperature. It shows the process of thermal convection [11]. The mechanism of thermogravitational (buoyancy-driven) convection and thermocapillary (surface tension driven) convection with temperature has been the subject of a great deal of theoretical and experimental investigations since the pioneering pieces of work of Rayleigh in 1916 [12] and Pearson in 1958 [13]. The analysis of thermogravitational and thermocapillary convection, respectively, has many important applications in the field of engineering such as flying of airplanes and submarine in physical engineering and production of colloids, paints, and polymeric suspensions in chemical engineering [14]. Rayleigh-Bénard instability is a mechanism associated with buoyancy since there appears a nondimensional Rayleigh 
number $R$, whereas Bénard-Marangoni instability is a mechanism associated with surface tension, since there appears a nondimensional Marangoni number $M$ [15]. Nield [16] has combined both mechanisms into a single analysis and the depth of the layer is found to decrease as the surface tension mechanism becomes more dominant, and when the depth of the layer is at most $0.1 \mathrm{~cm}$, the buoyancy effect can safely be neglected for most liquids. The pioneering pieces of work of Pearson, Rayleigh, Bénard, and Nield have subsequently been extended by many researchers such as Idris et al. [4], Siddheshwar and Pranesh [17], and Pranesh and Kiran [18].

The Rayleigh-Bénard-Marangoni convection in Eringen's micropolar fluid involves the effects of buoyancy and surface tension. The main result from all these studies can classify marginal states into two classes which are stationary and oscillatory states [19]. Stationary convection is the preferred mode for heating from below. Theoretically, oscillatory convection is the most possible mode for heating from above [17]. The theory of micropolar fluid is due to Eringen whose theory allows for the presence of particles in the fluid by taking into account particle motion and thermal effects [18]. We have indicated several possible applications of the theory to suspensions which are liquid crystals, polymeric fluids, certain anisotropic fluids, animal blood, and turbulence [14, 20].

Practically, considering uniformly distributed internal heat source for the problem is possible, which means having nonuniform basic temperature gradients [17]. In addition, various numbers of controllers are added up to examine whether their present will enhance or delay the onset of convection $[6,21]$.

The aim of this paper is to examine the effect of cubic temperature gradients with controller on the onset of RayleighBénard-Marangoni in electroconvection. The critical points for the onset of convection can be determined by using the Galerkin technique.

\section{Mathematical Formulation}

An infinite horizontal layer of a Boussinesquian micropolar fluid layer of depth $d$ is considered with the presence of an electric field. Take a Cartesian coordinate system $(x, y, z)$ with origin in the lower boundary and $z$-axis vertically upwards. The body forces acting on the fluid are buoyancy, surface tension, feedback control, and electric field. Furthermore, the spin is assumed to vanish at the boundaries.

The governing equations for the problem are the continuity equation, conservation of linear momentum, conservation of angular momentum, conservation of energy, equation of state, equation of state for dielectric constant, Faraday's law, and equation of polarization field; see [1].

Consider

$$
\begin{gathered}
\nabla \cdot \vec{q}=0 \\
\rho_{o}\left[\frac{\partial \vec{q}}{\partial t}+(\vec{q} \cdot \nabla) \vec{q}\right]=-\nabla p-\rho g \widehat{k}+(2 \zeta+\eta) \nabla^{2} \vec{q} \\
+\zeta(\nabla \times \vec{\omega})+(\vec{p} \cdot \nabla) \vec{E}
\end{gathered}
$$

$$
\begin{gathered}
\left.\rho_{o} I \frac{\partial \vec{\omega}}{\partial t}+(\vec{q} \cdot \nabla) \vec{\omega}\right]=\left(\lambda^{\prime}+\eta^{\prime}\right) \nabla(\nabla \cdot \vec{\omega})+\left(\eta^{\prime} \nabla^{2} \vec{\omega}\right) \\
+\zeta(\nabla \times \vec{q}-2 \vec{\omega}) \\
\frac{\partial T}{\partial t}+\left(\vec{q}-\frac{\beta}{\rho_{o} C_{v}} \nabla \times \vec{\omega}\right) \cdot \nabla T=\chi \nabla^{2} T \\
\rho=7 \rho_{o}\left[1-\alpha\left(T-T_{o}\right)\right] \\
\varepsilon_{r}=\left(1+\chi_{e}\right)-e\left(T-T_{o}\right) \\
\nabla \times \vec{E}=0, \quad \vec{E}=-\nabla \phi \\
\nabla \cdot\left(\varepsilon_{o} \vec{E}+\vec{P}\right)=0 \\
\vec{P}=\varepsilon_{o}\left(\varepsilon_{r}-1\right) \vec{E}
\end{gathered}
$$

where $\vec{q}$ is the velocity, $\vec{\omega}$ is the spin, $T$ is the temperature, $p$ is the hydrodynamic pressure, $\rho$ is the density, $\rho_{o}$ is the density of the fluid at reference temperature $T=T_{o}, g$ is the acceleration due to gravity, $\zeta$ is the coupling viscosity coefficient or vortex viscosity, $\eta$ is the shear kinematic viscosity coefficient, $I$ is the moment of inertia, $\lambda^{\prime}$ and $\eta^{\prime}$ are the bulk and shear spin viscosity coefficients, $\beta$ is the micropolar heat conduction coefficient, $C_{v}$ is the specific heat, $\chi$ is the thermal conductivity, $\alpha$ is the coefficient of thermal expansion, $\chi_{e}$ is thermal susceptibility, $\phi$ is the electrostatic potential, $\varepsilon_{o}$ is electric permeability of free space, $\varepsilon_{r}$ is dielectric constant, $\vec{P}$ is dielectric polarization, and $\vec{E}$ is electric field. The perturbation of (1) is nondimensionalised using the definition below:

$$
\begin{aligned}
& \left(x^{*}, y^{*}, z^{*}\right)=\frac{(x, y, z)}{d}, \quad W^{*}=\frac{W^{\prime}}{\chi / d}, \\
& \vec{\omega}^{*}=\frac{\omega^{\prime}}{\chi / d}, \quad t^{*}=\frac{t}{d^{2} / \chi},
\end{aligned}
$$

Following the classical lines of linear stability analysis as presented by Chandrasekhar [19], the linearised and dimensionless equations are given by

$$
\begin{gathered}
R \nabla_{1}^{2} T+\left(1+N_{1}\right) \nabla^{4} W+N_{1} \nabla^{2} \Omega_{z}+L \nabla_{1}^{2} T f(z) \\
-L \frac{\partial}{\partial z}\left(\nabla_{1}^{2} \phi\right) f(z)=0 \\
N_{3} \nabla^{2} \Omega_{z}-N_{1} \nabla^{2} W-2 N_{1} \Omega_{z}=0 \\
\nabla^{2} T+\left(W-N_{5} \Omega_{z}\right) f(z)=0 \\
\nabla^{2} \phi-\frac{\partial T}{\partial z}=0
\end{gathered}
$$


where $W, G, T$, and $\phi$ are, respectively, the amplitudes of the infinitesimal perturbations of velocity, spin, temperature, and electrostatic potential with

$$
\begin{gathered}
R=\frac{\rho_{0} \alpha g \Delta T d^{3}}{\chi(\zeta+\eta)}, \quad(\text { Ray leigh number }), \\
L=\frac{\varepsilon_{0} e^{2} E_{0}^{2} \Delta T^{2} d^{2}}{\left(1+\chi_{e}\right)(\zeta+\eta) \chi}, \quad(\text { Electric number }) \\
N_{1}=\frac{\zeta}{\zeta+\eta}, \quad\left(0 \leq N_{1} \leq 1\right), \quad(\text { Coupling parameter }) \\
N_{3}=\frac{\eta^{\prime}}{(\zeta+\eta) d^{2}}, \quad\left(0 \leq N_{3} \leq m\right) \\
N_{5}=\frac{\beta}{\rho_{0} C_{v} d^{2}}, \quad\left(0 \leq N_{5} \leq n\right),
\end{gathered}
$$

(Micropolarheat conduction parameter).

The infinitesimal perturbations $W, G, T$, and $\phi$ are assumed to be periodic waves and hence these permit a normal mode in the form [19]

$$
\begin{aligned}
& {\left[W, \Omega_{z}, T, \phi\right]} \\
& \quad=[W(z), G(z), T(z), \phi(z)] \exp [i(l x+m y)],
\end{aligned}
$$

where $l$ and $m$ are, respectively, the horizontal wave components of wave number $\vec{a}$. Equation (5) substituted into (3) yields

$$
\begin{gathered}
\left(1+N_{1}\right)\left(D^{2}-a^{2}\right)^{2} W-R a^{2} T+N_{1}\left(D^{2}-a^{2}\right) G \\
-L a^{2} T f(z)+L a^{2} D \phi(f(z))=0 \\
N_{3}\left(D^{2}-a^{2}\right) G-N_{1}\left(D^{2}-a^{2}\right) W-2 N_{1} G=0 \\
\left(D^{2}-a^{2}\right) T+\left(W-N_{5} G\right) f(z)=0 \\
\left(D^{2}-a^{2}\right) \phi-D T=0 .
\end{gathered}
$$

If $\zeta=0$, the above Rayleigh number identifies itself with the classical definition [17]. The operator $D=d / d z$ is defined as differentiation with respect to $z$ and $a$ is the dimensionless wave number [1].

Assume that the layer is bounded below by a rigid conducting isothermal surface and above by a free insulating adiabatic surface. Furthermore, the spin-boundary condition is assumed to vanish at the boundaries. Equations (6)-(9) are solved subject to the following boundary combinations:

$$
\begin{gathered}
W=D W=T=G=0 \quad \text { at } z=0, \\
W=D^{2} W+a^{2} M T=D T=G=0 \quad \text { at } z=1 .
\end{gathered}
$$

The nondimensional form $f(z)$ in (6) and (8) is given by

$$
f(z)=a_{1}^{*}+2 a_{2}^{*}(z-1)+3 a_{3}^{*}(z-1)^{2} .
$$

TABLE 1: Reference steady-state temperature gradients.

\begin{tabular}{lccccc}
\hline Model & $\begin{array}{c}\text { Reference } \\
\text { steady-state } \\
\text { temperature } \\
\text { gradients }\end{array}$ & $f(z)$ & $a_{1}^{*}$ & $a_{2}^{*}$ & $a_{3}^{*}$ \\
\hline 1 & Linear & 1 & 1 & 0 & 0 \\
2 & Cubic 1 & $3(z-1)^{2}$ & 0 & 0 & 1 \\
3 & Cubic 2 & $0.6+1.02(z-1)^{2}$ & 0.6 & 0 & 0.34 \\
\hline
\end{tabular}

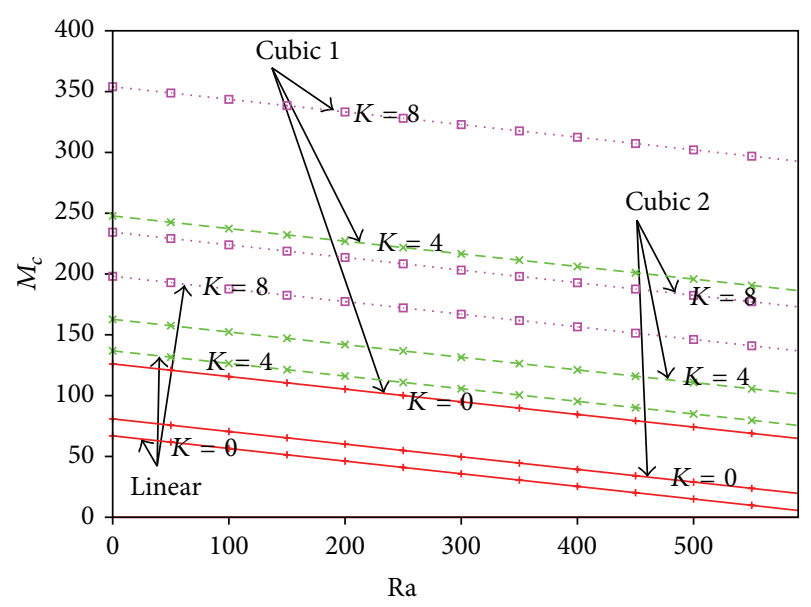

FIgURE 1: Plot of $M_{c}$ versus Ra for different values of $K=0, K=2$, and $K=4$.

The classical linear basic state temperature gradient is $a_{1}^{*}=1, a_{2}^{*}=0$, and $a_{3}^{*}=0$. The other different reference steady-state temperature gradients examined in this paper are listed in Table 1.

\section{Solution}

The Euler-Lagrange equations which are (6)-(9) are solved subject to the boundary conditions (10) which constitute an eigenvalue problem. The eigenvalue problem is solved by using the single-term Galerkin technique. The variables in (6)-(9) written in terms of trial functions which are $W_{1}, G_{1}$, $T_{1}$, and $\phi_{1}$ are related to $W, G, T$, and $\phi$ by $A=A W_{1}, G=B G_{1}$, $T=C T_{1}$, and $\phi=E \phi_{1}$, where $A, B, C$, and $E$ are constants, respectively. Equations (6)-(9) are multiplied by $W, G, T$, and $\phi$, respectively; then the equations are integrated with respect to $z$ from 0 to 1 and all the constants which are $A, B$, $C$, and $E$ are eliminated from the resulting equations. Lastly, the boundary conditions (10) are applied into the resulting equations. Here, we obtained the Marangoni number, $M$ :

$$
\begin{aligned}
M= & \left(R a^{2}\left\langle W_{1} T_{1}\right\rangle C_{3}-C_{2}\right. \\
& \times\left[\left\langle T_{1}\left(D^{2}-a^{2}\right) T_{1}\right\rangle+K T(1)\right] \\
& \left.+L a^{2} C_{3} C_{4}\right) \\
& \times\left(\left(1+N_{1}\right) a^{2} T(1) D W(1) C_{3}\right)^{-1},
\end{aligned}
$$




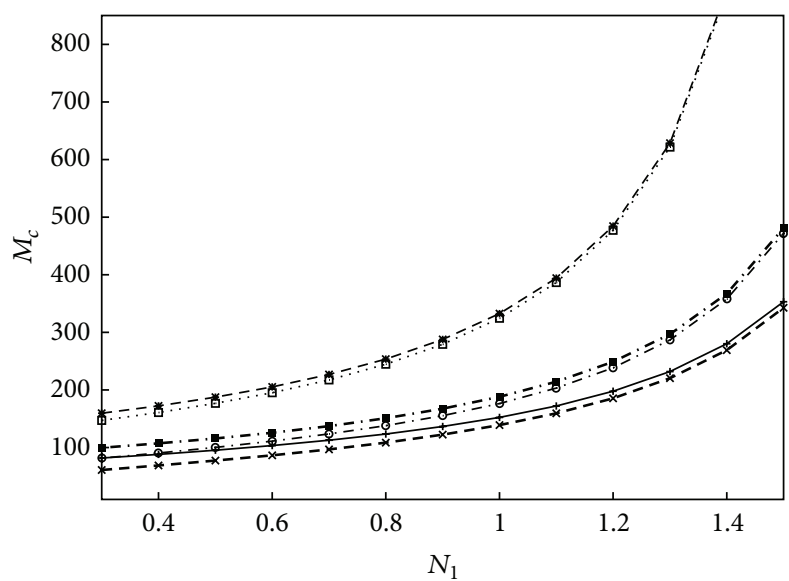

— Linear, $L=100$

-*- Linear, $L=300$

-*-Cubic $1, L=100$

ロ. Cubic $1, L=300$

$\rightarrow-$ Cubic 2, $L=100$

- - - Cubic 2, $L=300$

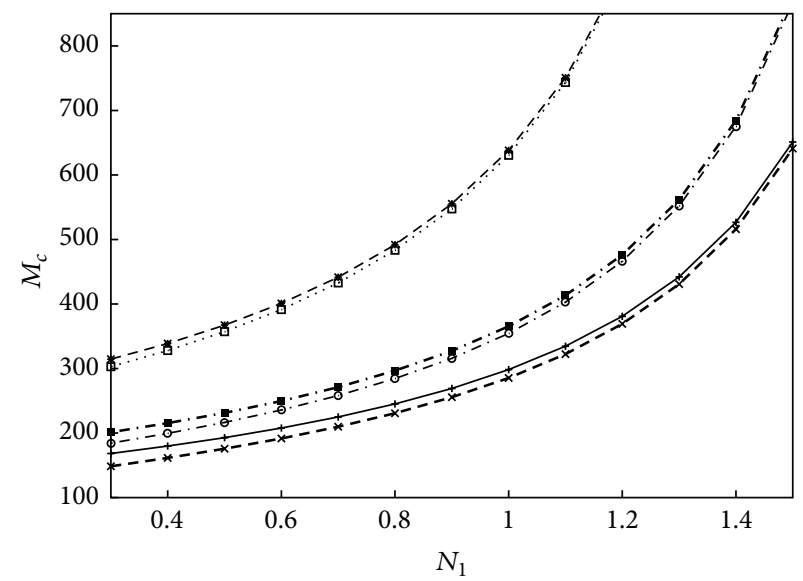

(a)

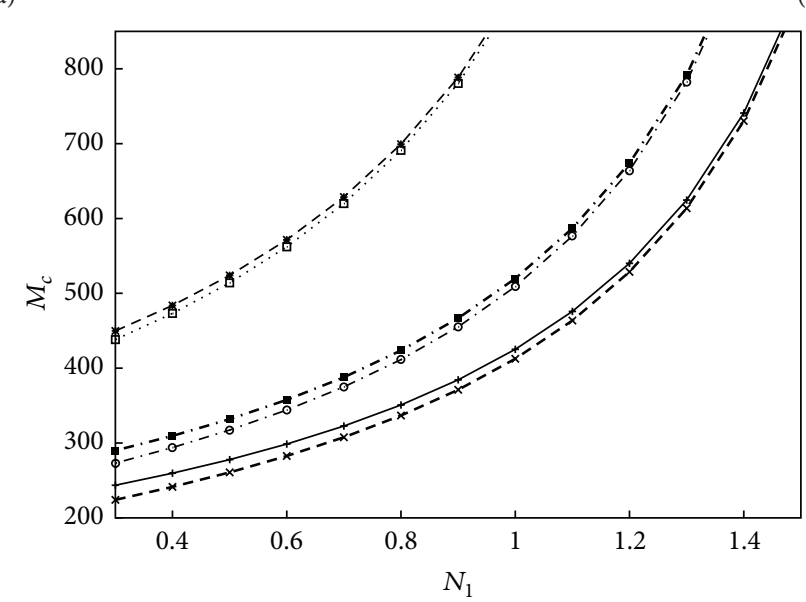

— Linear, $L=100$
-*- Linear, $L=300$
*- Cubic $1, L=100$

•ロ. Cubic $1, L=300$

$\rightarrow-$ Cubic 2, $L=100$

$\rightarrow-$ Cubic 2, $L=300$

(b) $\longrightarrow$ Linear, $L=100$

- * - Linear, $L=300$

-* - Cubic 1, $L=100$

$$
\begin{aligned}
& \text { • } \square \text {.. Cubic } 1, L=300 \\
& \rightarrow \text { - Cubic 2, } L=100 \\
& \text { - }- \text { - Cubic 2, } L=300
\end{aligned}
$$

(c)

Figure 2: Plot of critical Marangoni number, $M_{c}$, versus couple parameter, $N_{1}$, for different electric number, $L$, and (a) $K=0$, (b) $K=4$, and (c) $K=8$.

where

$$
\begin{aligned}
C_{1}= & N_{3}\left\langle G_{1}\left(D^{2}-a^{2}\right) G_{1}\right\rangle-2 N_{1}\left\langle G_{1}^{2}\right\rangle, \\
C_{2}= & \left(1+N_{1}\right)\left\langle W_{1}\left(D^{2}-a^{2}\right)^{2} W_{1}\right\rangle C_{1} \\
& +N_{1}^{2}\left\langle W_{1}\left(D^{2}-a^{2}\right) G_{1}\right\rangle\left\langle G_{1}\left(D^{2}-a^{2}\right) W_{1}\right\rangle, \\
C_{3}= & N_{1} N_{5}\left\langle G_{1}\left(D^{2}-a^{2}\right) W_{1}\right\rangle\left\langle T_{1} G_{1} f(z)\right\rangle \\
& -C_{1}\left\langle T_{1} W_{1} f(z)\right\rangle, \\
& \\
C_{4}= & \left\langle W_{1} T_{1} f(z)\right\rangle-\frac{\left\langle W_{1} D \phi_{1} f(z)\right\rangle\left\langle\phi_{1} D T_{1}\right\rangle}{\left\langle\phi_{1}\left(D^{2}-a^{2}\right) \phi_{1}\right\rangle},
\end{aligned}
$$

where the angle brackets \langle\rangle denote the integration with respect to $z$ when $z$ is between 0 and 1 . We choose the trial functions for lower rigid isothermal and upper free adiabatic as follows:

$$
\begin{gathered}
W_{1}=z^{4}-\frac{5}{2} z^{3}+\frac{3}{2} z^{2}, \\
T_{1}=z(2-z), \quad G_{1}=z(1-z), \\
\phi_{1}=z^{2}(3-2 z)
\end{gathered}
$$

such that they all satisfy the boundary conditions in (5) and (6), except the one given by $D^{2} W+a^{2} M T=0$ at $z=1$, but the residual from this is included in the residual from the differential equations. 


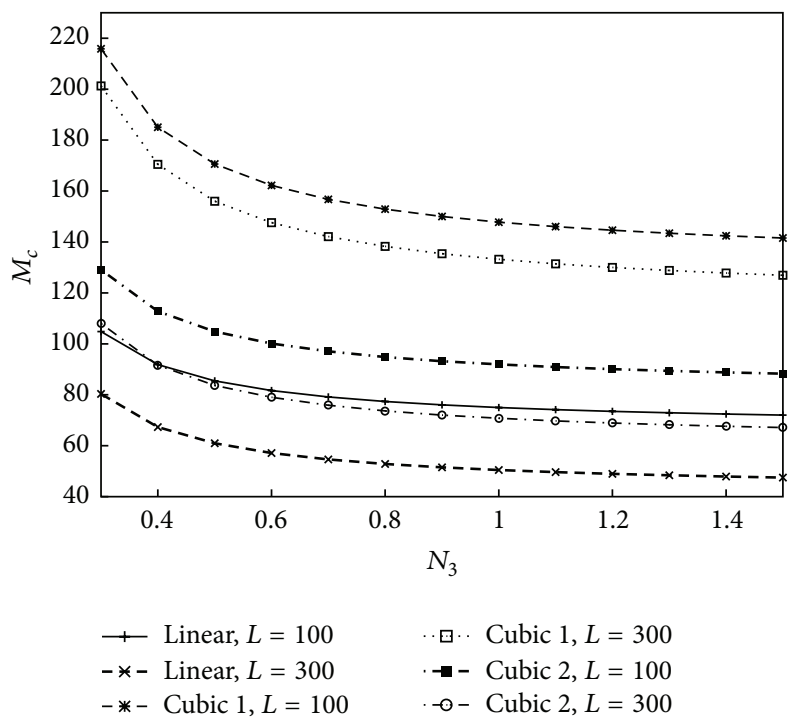

(a)

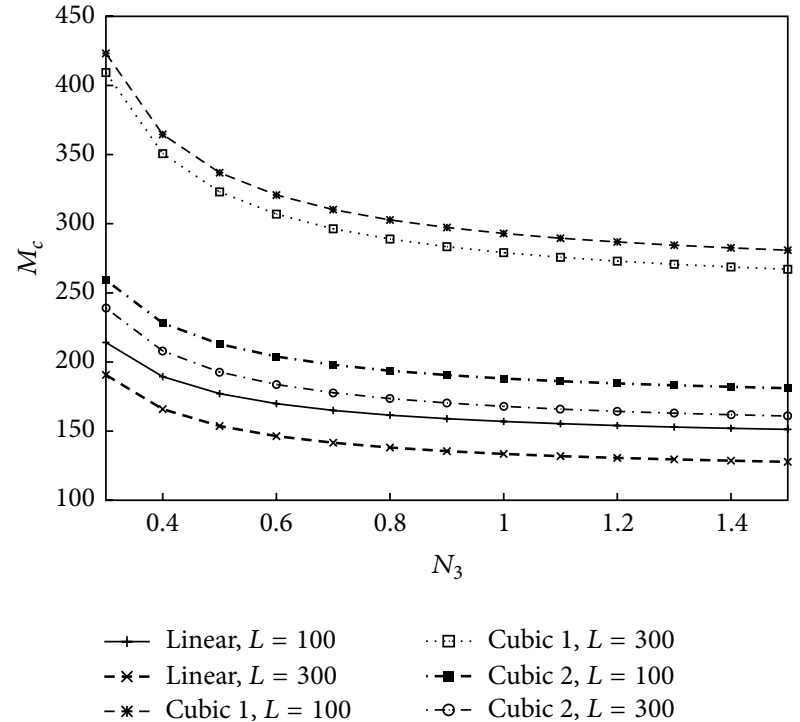

(b)

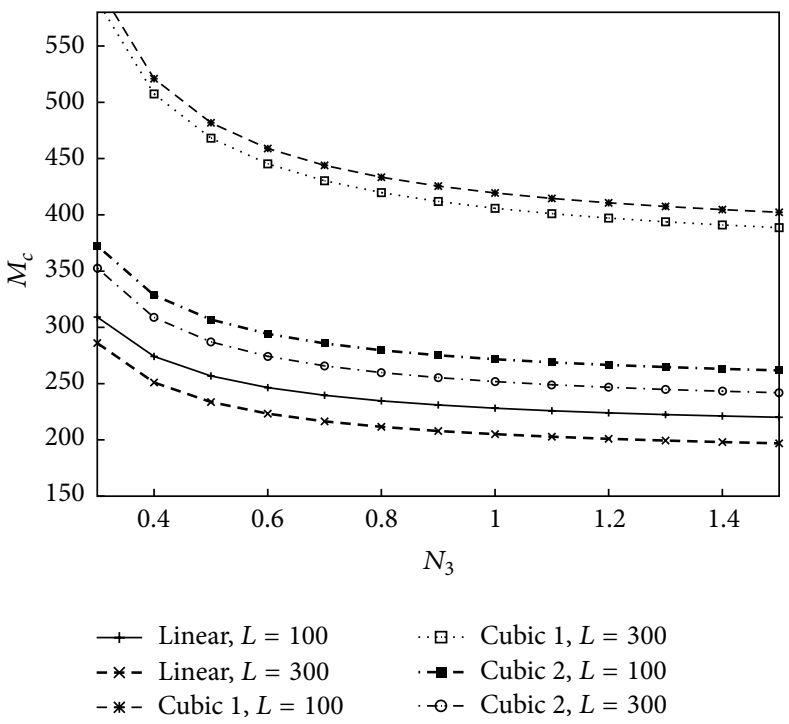

(c)

Figure 3: Plot of critical Marangoni number, $M_{c}$, versus couple stress parameter, $N_{3}$, for different electric number, $L$, and (a) $K=0$, (b) $K=4$, and (c) $K=8$.

\section{Results and Discussion}

The aim of this paper is to study the effects of feedback control $[6,21]$ together with the effect of nonuniform temperature profiles on the onset of Rayleigh-Bénard-Marangoni [1, 4, 22, 23] electroconvection.

The graph of the critical Marangoni number $M_{c}$ as a function of Rayleigh number, Ra, for the different values of feedback control, $K$, is plotted with different temperature profiles as in Figure 1. From Figure 1, we concluded that, for the different temperature profiles, increasing value of Rayleigh number, Ra, decreased the critical Marangoni number, $M_{c}$, significantly; hence, the system becomes more unstable. The onset of convection will be delayed when the value of feedback control, $K$, in the system is increased. As the feedback control, $K$, gains, $M_{c}$ increases monotonically, showing that the feedback control stabilizes the no-motion state for all wave numbers. Meanwhile, we observed that the presence of buoyancy force, $\mathrm{Ra}$, promoted the onset of convection. Our results showed that Model 1 (Linear) with $a_{1}^{*}=1, a_{2}^{*}=0$, and $a_{3}^{*}=0$ is less stabilizing than Model 3 (Cubic 2); that is, $M_{c 3}<M_{c 1}$. Further, the results indicate that Model 2 (Cubic 1) with $a_{1}^{*}=0, a_{2}^{*}=0$, and $a_{3}^{*}=1$ is the most stabilizing of all the considered types of temperature gradients; that is, $M_{c 1}<M_{c 3}<M_{c 2}$.

Figures 2(a)-2(c) illustrate the critical Marangoni number, $M_{c}$, versus coupling parameter, $N_{1}$, for the different values of feedback control, $K$, and electric number, $L$, 


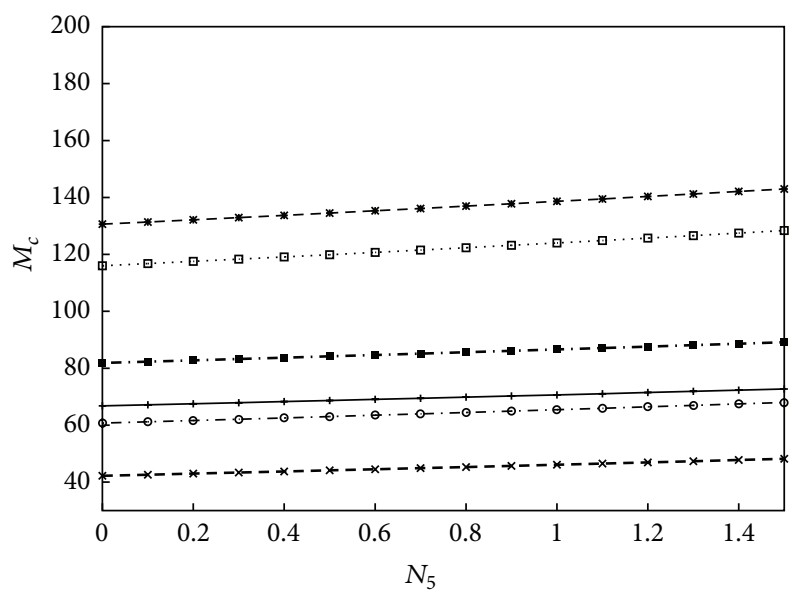

1 Linear, $L=100$

-*- Linear, $L=300$

-*- Cubic $1, L=100$

(a)

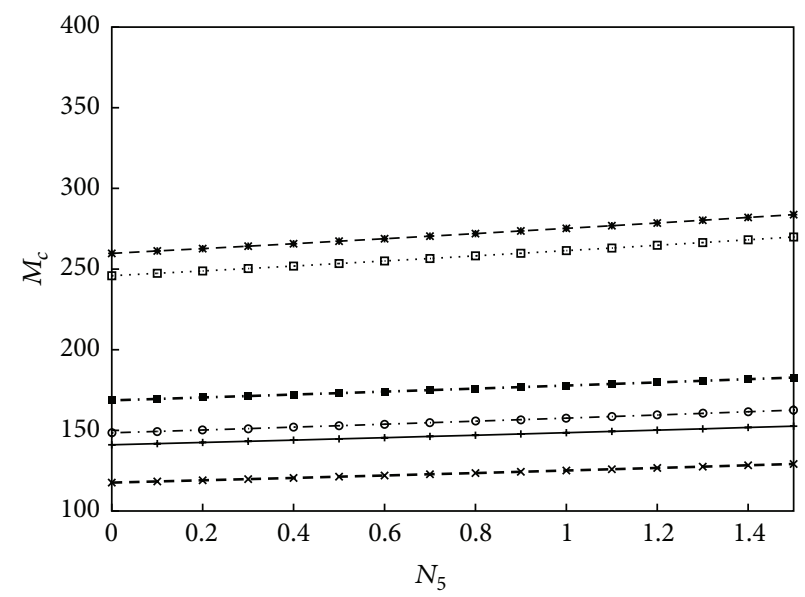

। Linear, $L=100$

-*- Linear, $L=300$

-*- Cubic $1, L=100$

๑. Cubic $1, L=300$

$\rightarrow-$ Cubic $2, L=100$

- - - Cubic $2, L=300$

(b)

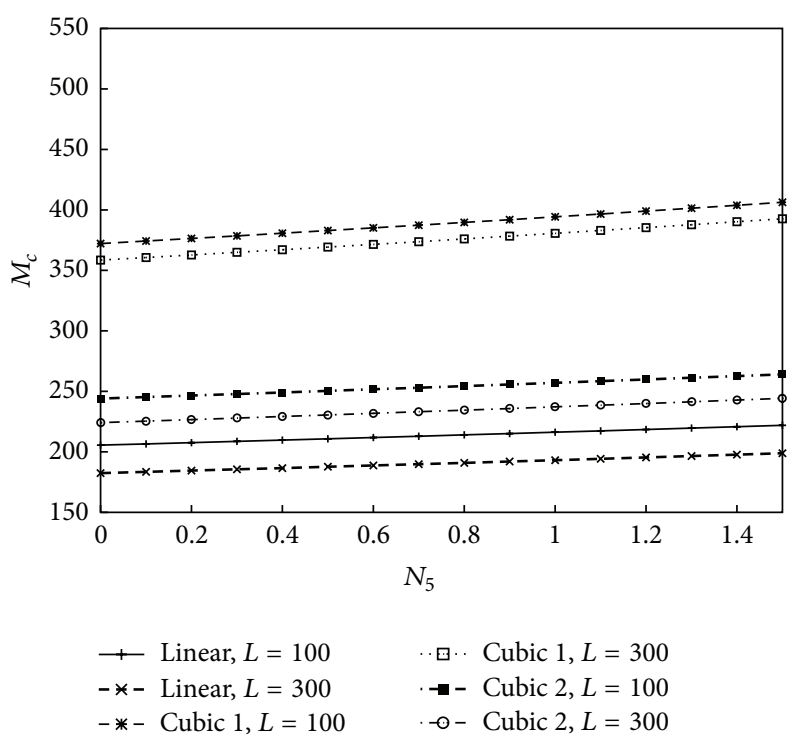

(c)

Figure 4: Plot of critical Marangoni number, $M_{c}$, versus micropolar heat conduction parameter, $N_{5}$, for different electric number, $L$, and (a) $K=0$, (b) $K=4$, and (c) $K=8$.

is plotted with different temperature profiles for lower rigid isothermal and upper free adiabatic boundaries. Clearly, $M_{c}$ increases with $N_{1}$. Increases in $N_{1}$ indicate the increasing concentration of microelements. As microelements increase in number with $N_{1}$, greater part of the energy system is consumed by these elements in developing the gyrational velocities of the fluid and it concludes that onset of convection is delayed. Increasing $N_{1}$ is to stabilize the system. From Figure 2, it is also observed that the increase in electric number, $L$, decreases the critical Marangoni number, $M_{c}$; as a result, the onset of convection is delayed. Therefore, the electric number, $L$, destabilizes the system. Besides, we have examined that the increasing value of $K$ delayed the onset of convection and rendered the system stable. From those three temperature gradients, Model 2 (Cubic 1 ) is shown to be the most stabilizing with $a_{1}^{*}=0, a_{2}^{*}=0$, and $a_{3}^{*}=1$ of all the considered types of the temperature gradients; that is, $M_{c 1}<M_{c 3}<M_{c 2}$.

Figures 3(a)-3(c) illustrate the critical Marangoni number, $M_{c}$, versus couple stress parameter, $N_{3}$, for the different values of feedback control, $K$, and electric number, $L$, in the absence of buoyancy force, $\mathrm{Ra}$, is plotted with different temperature profiles. From Figure 3, $M_{c}$ decreases with increasing $N_{3}$. Increasing $N_{3}$ decreases the couple stress of the fluid which causes a decreasing in microrotation and hence makes the system more unstable. Thus, increasing $N_{3}$ destabilizes the system as couple stresses are operative at only small values of $N_{3}$. Decrease in $M_{c}$ is significant for lower 
values of $N_{3}$; at higher values, dip in $M_{c}$ is insignificant. Meanwhile, the onset of convection would be delayed when the value of feedback control, $K$, in the system is increased. Linear temperature profile is less stabilizing than Cubic 2, and Cubic 1 is the most stabilizing one; that is, $M_{c 1}<M_{c 3}<M_{c 2}$. Besides, it illustrates that increasing electric number, $L$, will decrease critical Marangoni number, $M_{c}$; as a result, the onset of convection is delayed.

Figures 4(a)-4(c) illustrate the results of the critical Marangoni number, $M_{c}$, versus micropolar heat conduction parameter, $N_{5}$, for the different values of feedback control, $K$, and electric number, $L$, is plotted with different temperature profiles gradient. When $N_{5}$ increases, $M_{c}$ also increases. Increasing micropolar heat conduction parameter, $N_{5}$, will make the heat induced into the fluid due to these microelements also increase, thus reducing the heat transfer from bottom to the top. Decrease in heat transfer will delay the onset of instability. $N_{5}$ acts as a stabilizing effect on the system. Meanwhile, increases in feedback control, $K$, for every temperature gradient will delay the onset of convection in the system. The different temperature gradients are Linear, Cubic 1, and Cubic 2. Cubic 1 is the most stabilizing one among them. Linear temperature profile is less stabilizing compared to Cubic 2; that is, $M_{c 1}<M_{c 3}<M_{c 2}$. It is also observed that the increase in electric number, $L$, decreases the critical Marangoni number, $M_{c}$. As a result, the onset of convection is delayed. Therefore, electric number, $L$, acts as a destabilizing effect on the system.

\section{Conclusion}

In this work, we used the classical linear stability analysis to investigate the effect of feedback control, electric number, and different temperature gradients profiles on the onset of thermocapillary and thermogravitational convection in a dielectric micropolar fluid. Our results provided evidence that the presence of feedback control helped in delaying the onset of convection in dielectric micropolar fluid. When the value of feedback control increased, then the system became more stable. Electric number which acts as a destabilizing effect on the system will delay the onset of thermal convection. Of the three models considered in this paper, Model 2 (Cubic 1) is the most stable compared to Model 1 (Linear) and Model 3 (Cubic 2). Model 3 (Cubic 2) is suitable for consideration for laboratory experimentation with microgravity environment. Therefore, we concluded that dielectric field mechanism has a destabilizing effect on the system.

\section{Conflict of Interests}

The authors declare that there is no conflict of interests regarding the publication of this paper.

\section{Acknowledgments}

The authors acknowledge the financial support received from the Universiti Malaysia Terengganu under Grant
UMT-TPM and the Ministry of Education Malaysia for MyBrain Program.

\section{References}

[1] S. Pranesh and R. Baby, "Effect of non-uniform temperature gradient on the onset of Rayleigh-Bénard electro convection in a micropolar fluid," Applied Mathematics, vol. 3, no. 5, pp. 442450, 2012.

[2] P. H. Roberts, "Electrohydrodynamic convection," Quarterly Journal of Mechanics and Applied Mathematics, vol. 22, no. 2, pp. 211-220, 1969.

[3] M.-I. Char and K.-T. Chiang, "Boundary effects on the BénardMarangoni instability under an electric field," Applied Scientific Research, vol. 52, no. 4, pp. 331-354, 1994.

[4] R. Idris, H. Othman, and I. Hashim, "On effect of non-uniform basic temperature gradient on Bénard-Marangoni convection in micropolar fluid," International Communications in Heat and Mass Transfer, vol. 36, no. 3, pp. 255-258, 2009.

[5] M. N. Mahmud, R. Idris, and I. Hashim, "Effects of magnetic field and nonlinear temperature profile on Marangoni convection in micropolar fluid," Differential Equations and Nonlinear Mechanics, vol. 2009, Article ID 748794, 11 pages, 2009.

[6] R. Idris and I. Hashim, "Effects of controller and cubic temperature profile on onset of Bénard-Marangoni convection in ferrofluid," International Communications in Heat and Mass Transfer, vol. 37, no. 6, pp. 624-628, 2010.

[7] R. Idris and I. Hashim, "On the effects of a cubic temperature profile on oscillatory Rayleigh-Bénard convection in a viscoelastic fluid-filled high-porosity medium," Journal of Porous Media, vol. 14, no. 5, pp. 437-447, 2011.

[8] I. Tanasawa, "Experimental techniques in natural convection," Experimental Thermal and Fluid Science, vol. 10, no. 4, pp. 503518, 1995.

[9] A. Douiebe, M. Hannaoui, G. Lebon, A. Benaboud, and A. Khmou, "Effects of a.c. electric field and rotation on BénardMarangoni convection," Flow, Turbulence and Combustion, vol. 67, no. 3, pp. 185-204, 2002.

[10] M. F. El-Sayed, "Onset of electroconvective instability of Oldroydian viscoelastic liquid layer in Brinkman porous medium," Archive of Applied Mechanics, vol. 78, no. 3, pp. 211224, 2008.

[11] P. G. Drazin, Introduction to Hydrodynamic Stability, Cambridge Texts in Applied Mathematics, Cambridge University Press, Cambridge, UK, 2002.

[12] L. Rayleigh, "On convection currents in a horizontal layer of fluid when the higher temperature is on the under side," Philosophical Magazine Series 6, vol. 32, no. 192, pp. 529-546, 1916.

[13] J. R. A. Pearson, "On convection cells induced by surface tension," Journal of Fluid Mechanics, vol. 4, no. 5, pp. 489-500, 1958.

[14] A. C. Eringen, “Theory of micropolar fluids," Journal of Mathematics and Mechanics, vol. 16, pp. 1-35, 1966.

[15] A. K. Gupta and R. G. Shandil, "Marangoni convection in a relatively hotter or cooler liquid layer with free boundaries," International Journal of Applied Mechanics and Engineering, vol. 18, no. 2, pp. 581-588, 2013.

[16] D. A. Nield, "Surface tension and buoyancy effects in cellular convection," Journal of Fluid Mechanics, vol. 19, pp. 341-352, 1964. 
[17] P. G. Siddheshwar and S. Pranesh, "Effect of a non-uniform basic temperature gradient on Rayleigh-Benard convection in a micropolar fluid," International Journal of Engineering Science, vol. 36, no. 11, pp. 1183-1196, 1998.

[18] S. Pranesh and R. V. Kiran, "Study of Rayleigh-Bénard Magneto convection in a micropolar fluid with Maxwell-Cattaneo law," Applied Matehmatics, vol. 1, no. 1, pp. 470-480, 2010.

[19] S. Chandrasekhar, Hydrodynamic and hydromagnetic stability, The International Series of Monographs on Physics, Clarendon Press, Oxford, 1961.

[20] A. C. Eringen, "Theory of thermomicrofluids," Journal of Mathematical Analysis and Applications, vol. 38, no. 2, pp. 480496, 1972.

[21] M. N. Mahmud, Z. Mustafa, and I. Hashim, "Effects of control on the onset of Bénard-Marangoni convection in a micropolar fluid," International Communications in Heat and Mass Transfer, vol. 37, no. 9, pp. 1335-1339, 2010.

[22] I. S. Shivakumara, N. Rudraiah, and C. E. Nanjundappa, "Effect of non-uniform basic temperature gradient on RayleighBenard-Marangoni convection in ferrofluids," Journal of Magnetism and Magnetic Materials, vol. 248, no. 3, pp. 379-395, 2002.

[23] T. D. Foster, "Effect of boundary conditions on the onset of convection," Physics of Fluids, vol. 11, no. 6, pp. 1257-1262, 1968. 


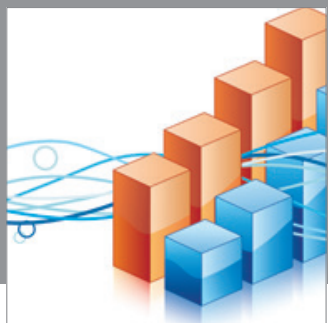

Advances in

Operations Research

mansans

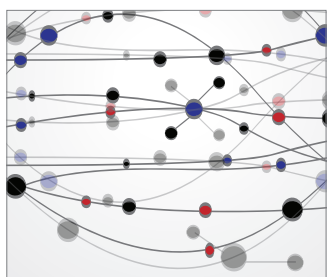

The Scientific World Journal
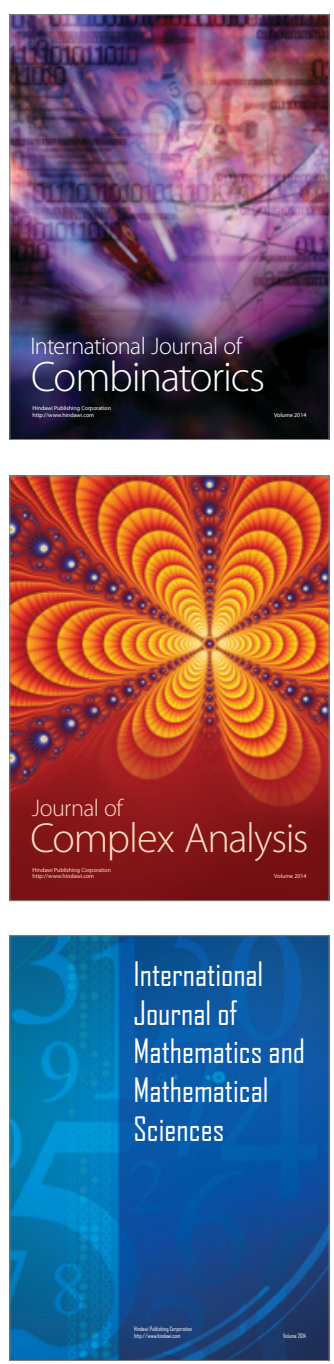
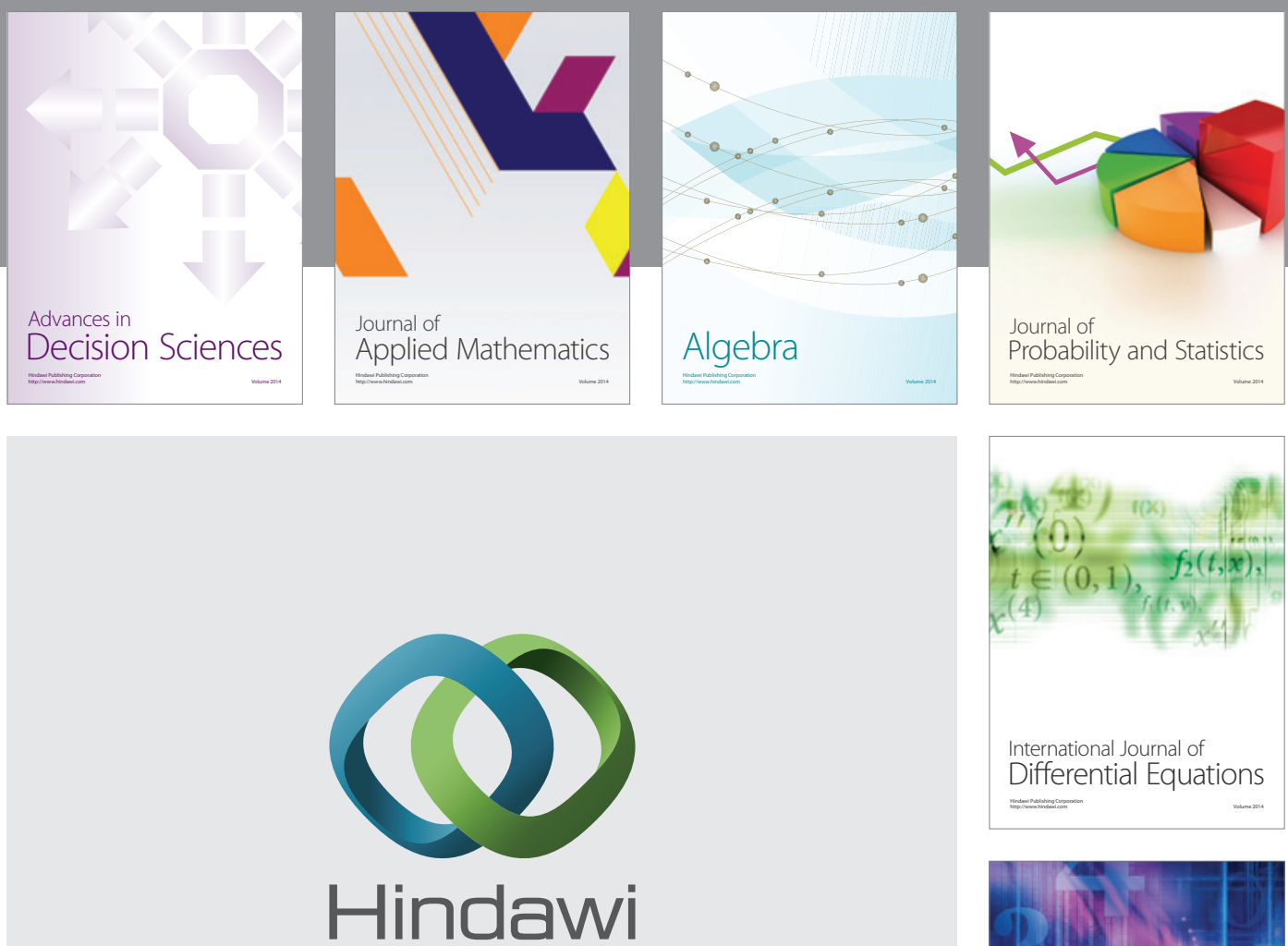

Submit your manuscripts at http://www.hindawi.com
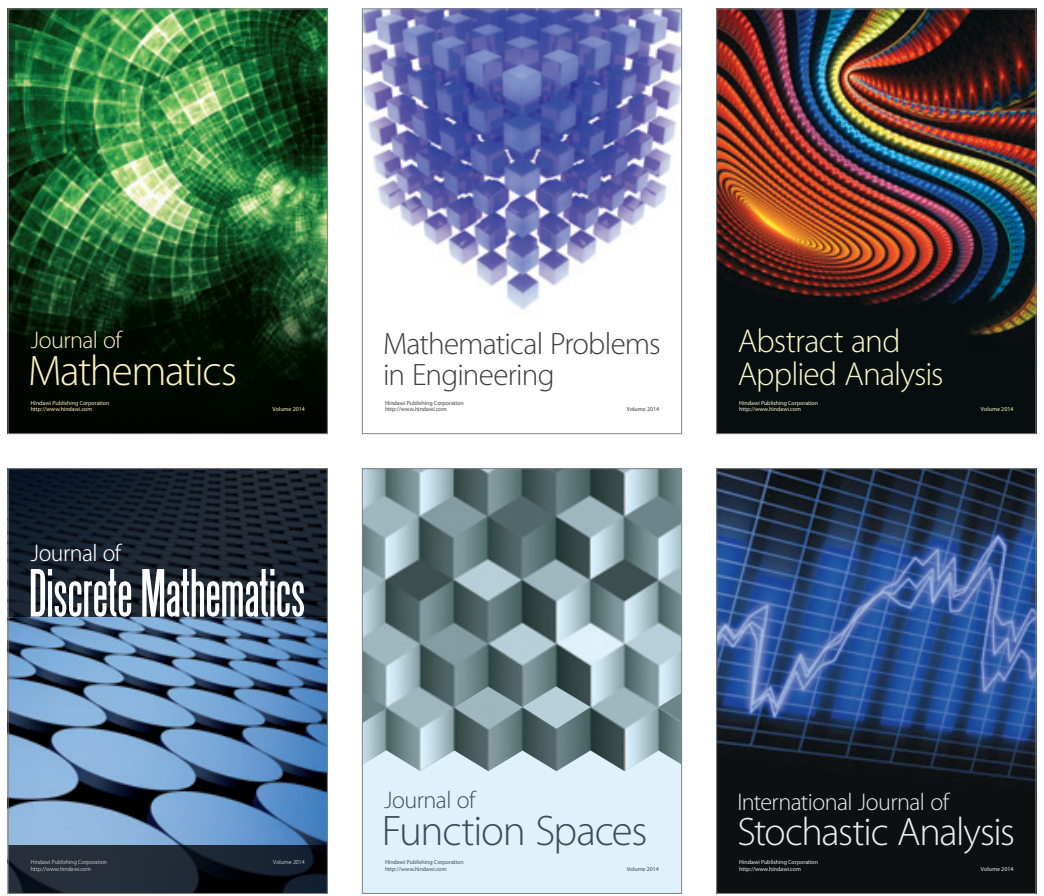

Journal of

Function Spaces

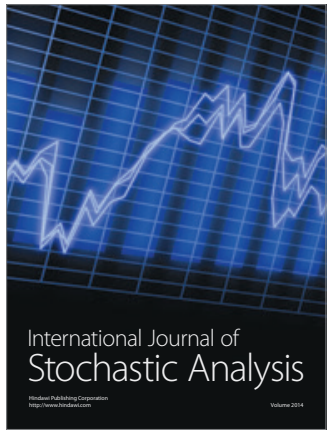

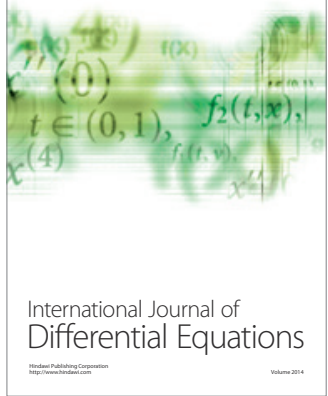
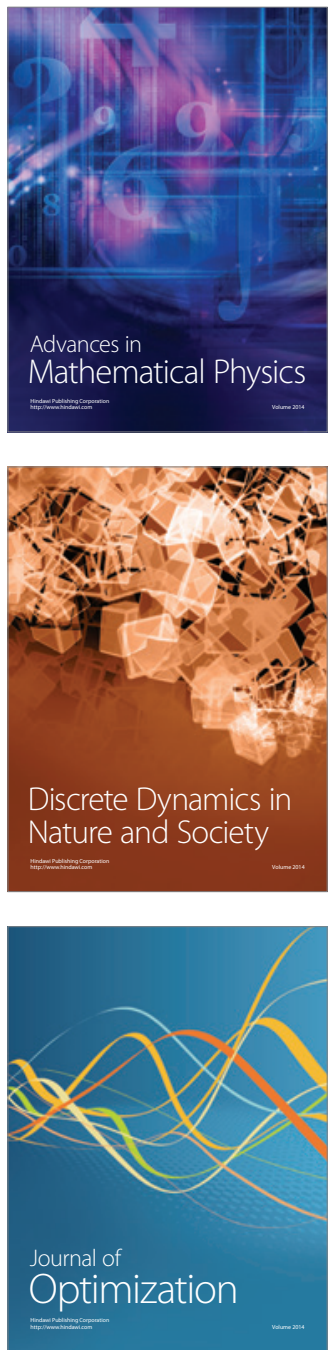